International Journal of Pure and Applied Mathematics

Volume 92 No. 4 2014, 591-597

ISSN: 1311-8080 (printed version); ISSN: 1314-3395 (on-line version)

url: http://www.ijpam.eu

doi: http://dx.doi.org/10.12732/ijpam.v92i4.14

\title{
NEIGHBORHOOD OF A CERTAIN FAMILY \\ OF MULTIVALENT FUNCTIONS WITH \\ NEGATIVE COEFFICIENTS
}

\author{
Jamal Salah \\ Department of Basic Sciences \\ College of Applied Sciences \\ A'Sharqiya University \\ Ibra, A'Sharqiya, OMAN
}

\begin{abstract}
By the use of the integration fractional operator introduced by authors [11],we introduce and investigate two new subclasses of $p$-valently analytic functions of complex order.we obtain various results for each of these classes including coefficient inequalities and inclusion relationship involving the $(n, \delta)$-neighborhood of $p$-valenty analytic functions.
\end{abstract}

Key Words: analytic functions, $p$-valent functions, coefficient inequalities, inclusion relations, neighborhood properties, $(n, \delta)$-neighborhood

\section{Introduction}

Let $A_{p}(n)$ denote the class of all analytic functions $f(z)$ normalized by

$$
f(z)=z^{p}-\sum_{k=n+p}^{\infty} a_{k} z^{k},\left(a_{k} \geq 0 ; n, p \in N:=\{1,2,3, \ldots\}\right),
$$

which are analytic and $p$-valent in the open unit disk, $U=\{z:|z|<1\}$. Now

Received: February 26, 2014

(c) 2014 Academic Publications, Ltd. url: www.acadpubl.eu 
for $f(z) \in A_{p}(n)$,we have

$$
J_{\eta, \lambda} f(z)=\phi(p) z^{p}-\sum_{k=n+p}^{\infty} \phi(k) a_{k} z^{k}
$$

Here $J_{\eta, \lambda} f(z)$, is the integration operator defined by authors, see [1],

$$
\begin{aligned}
& \phi(k)=\frac{(\Gamma(k+1))^{2} \Gamma(2+\eta-\lambda) \Gamma(2-\eta)}{\Gamma(k+\eta-\lambda+1) \Gamma(k-\eta+1)}, \\
& \phi(p)=\frac{(\Gamma(p+1))^{2} \Gamma(2+\eta-\lambda) \Gamma(2-\eta)}{\Gamma(p+\eta-\lambda+1) \Gamma(p-\eta+1)},
\end{aligned}
$$

$(n, p \in N, \eta-1<\lambda \leq \eta<2)$. Making use of the function $J_{\eta, \lambda} f(z)$ given by (1.2), we introduce the following subclasses of $p$-valently analytic function class $A_{p}(n)$.

Definition 1.1. A function $f(z)$ defined by (1.1) is said to be in the class $J_{\eta, \lambda}^{P}(\gamma, b)$, if and only if:

$$
\left|\frac{1}{b}\left(\frac{z J_{\eta, \lambda}^{\prime} f(z)+\gamma z^{2} J_{\eta, \lambda}^{\prime \prime} f(z)}{\gamma z J_{\eta, \lambda}^{\prime} f(z)+(1-\gamma) J_{\eta, \lambda} f(z)}-p\right)\right|<1
$$

$(n, p \in N, \eta-1<\lambda \leq \eta<2, b \in C, 0 \leq \gamma \leq 1)$.

Definition 1.2. A function $f(z)$ defined by (1.1) is said to be in the class $G_{\eta, \lambda}^{P}(\gamma, b)$, if and only if:

$$
\left|\frac{1}{b}\left(J_{\eta, \lambda}^{\prime} f(z)+\gamma z J_{\eta, \lambda}^{\prime \prime} f(z)-p\right)\right|<p
$$

$(n, p \in N, \eta-1<\lambda \leq \eta<2, b \in C, 0 \leq \gamma \leq 1)$. Next, we define the $(n, \delta)$ neighborhood of a function $f(z) \in A_{p}(n)$ see $[2]$

$$
\begin{aligned}
& N_{n, \delta}(f) \\
& \quad:=\left\{g: g \in A_{p}(n), g(z)=z^{p}-\sum_{k=n+p}^{\infty} b_{k} z^{k} \text { and } \sum_{k=n+p}^{\infty} k\left|a_{k}-b_{k}\right| \leq \delta\right\} .
\end{aligned}
$$

It follows from (1.5) that, if

$$
h(z)=z^{p},(p \in N),
$$


Then

$$
N_{n, \delta}(h):=\left\{g: g \in A_{p}(n), g(z)=z^{p}-\sum_{k=n+p}^{\infty} b_{k} z^{k} \text { and } \sum_{k=n+p}^{\infty} k\left|b_{k}\right| \leq \delta\right\} .
$$

The main object of the present work is to investigate the various properties and characteristics of analytic $p$-valent functions belonging to the subclasses $J_{\eta, \lambda}^{P}(\gamma, b)$, and $G_{\eta, \lambda}^{P}(\gamma, b)$ which are introduced by making use of the operator $J_{\eta, \lambda} f(z)$ on the normalized $p$-valently analytic functions in $U$. Apart from deriving a set of coefficient inequalities for each of these two function classes, we establish some inclusion relationships involving the $(n, \delta)$-neighborhoods of analytic $p$-valent functions belonging to each of these classes.

\section{Coefficient Inequalities}

In this section, we prove the following results which yield the coefficient inequalities for functions in the subclasses $J_{\eta, \lambda}^{P}(\gamma, b)$, and $G_{\eta, \lambda}^{P}(\gamma, b)$.

Theorem 2.1. Let $f(z) \in A_{p}(n)$ be given by (1.1). Then $f(z) \in J_{\eta, \lambda}^{P}(\gamma, b)$ if and only if

$$
\begin{gathered}
\sum_{k=n+p}^{\infty}(k+|b|-p)[\gamma(k-1)+1] \phi(k) a_{k} \leq|b|[\gamma(p-1)+1] \phi(p) \\
\phi(k)=\frac{(\Gamma(k+1))^{2} \Gamma(2+\eta-\lambda) \Gamma(2-\eta)}{\Gamma(k+\eta-\lambda+1) \Gamma(k-\eta+1)} \\
\phi(p)=\frac{(\Gamma(p+1))^{2} \Gamma(2+\eta-\lambda) \Gamma(2-\eta)}{\Gamma(p+\eta-\lambda+1) \Gamma(p-\eta+1)}
\end{gathered}
$$

Proof. Let a function $f(z)$ of the form (1.1) belong to the class $J_{\eta, \lambda}^{P}(\gamma, b)$. Then, in view of (1.2) and (1.3), we obtain the following inequality:

$$
\Re\left(\frac{\sum_{k=n+p}^{\infty}(p-k)[\gamma(k-1)+1] \phi(k) a_{k} z^{k}}{[\gamma(p-1)+1] \phi(p) z^{p}-\sum_{k=n+p}^{\infty}[\gamma(k-1)+1] \phi(k) a_{k} z^{k}}\right)>-|b| .
$$

Setting $z=r(0 \leq r<1)$ in (2.2), we observe that the expression in the denominator on the left-hand side of (2.2) is positive for $r=0$ and also for 
all $(0<r<1)$. Thus by letting $z \rightarrow 1^{-}$through real values, $(2.2)$ leads us to desired assertion (2.1) of theorem 2.1. Conversely, by applying (2.1)and setting $|z|=1$, we find from (1.2) that

$$
\begin{aligned}
\left|\frac{z J_{\eta, \lambda}^{\prime} f(z)+\gamma z^{2} J_{\eta, \lambda}^{\prime \prime} f(z)}{\gamma z J_{\eta, \lambda}^{\prime} f(z)+(1-\gamma) J_{\eta, \lambda} f(z)}-p\right| & \left|\frac{\sum_{k=n+p}^{\infty}(p-k)[\gamma(k-1)+1] \phi(k) a_{k} z^{k}}{\mid \gamma(p-1)+1] \phi(p) z^{p}-\sum_{k=n+p}^{\infty}[\gamma(k-1)+1] \phi(k) a_{k} z^{k}}\right| \\
= & \left|\frac{|b|\left\{[\gamma(p-1)+1] \phi(p)-\sum_{k=n+p}^{\infty}[\gamma(k-1)+1] \phi(k) a_{k}\right\}}{[\gamma(p-1)+1] \phi(p)-\sum_{k=n+p}^{\infty}[\gamma(k-1)+1] \phi(k) a_{k}}=\right| b \mid .
\end{aligned}
$$

Hence, by the maximum modulus principle, we infer that $f(z) \in J_{\eta, \lambda}^{P}(\gamma, b)$, which completes the proof of Theorem 2.1.

Similarly, we can prove the following theorem.

Theorem 2.2. Let $f(z) \in A_{p}(n)$ be given by (1.1). Then $f(z) \in G_{\eta, \lambda}^{P}(\gamma, b)$ if and only if

$$
\sum_{k=n+p}^{\infty}[\gamma(k-1)+1] k \phi(k) a_{k} \leq p(|b|-1)+[\gamma(p-1)+1] p \phi(p) .
$$

\section{Inclusion Relations Involving the $(n, \delta)$-Neighborhoods}

In this section, we establish several inclusion relations for the normalized $p$ valently analytic function classes $J_{\eta, \lambda}^{P}(\gamma, b)$ and $G_{\eta, \lambda}^{P}(\gamma, b)$, involving the $(n, \delta)$ neighborhood defined in (1.7).

Theorem 3.1. If

$$
\delta:=\frac{|b|[\gamma(p-1)+1](n+p) \phi(p)}{(n+|b|)[\gamma(n+p-1)] \phi(n+p)},(p>|b|),
$$

then

$$
J_{\eta, \lambda}^{P}(\gamma, b) \subset N_{n, \delta}(h)
$$


Proof. Let $f(z) \in J_{\eta, \lambda}^{P}(\gamma, b)$. Then in view of Theorem 2.1, we have

$$
(n+|b|)[\gamma(n+p-1)+1] \phi(n+p) \sum_{k=n+p}^{\infty} a_{k} \leq|b|[\gamma(p-1)+1] \phi(p)
$$

which yields

$$
\sum_{k=n+p}^{\infty} a_{k} \leq \frac{|b|[\gamma(p-1)+1] \phi(p)}{(n+|b|)[\gamma(n+p-1)+1] \phi(n+p)}
$$

Now,using (2.1) and (3.4), we get $[\gamma(n+p-1)+1] \phi(n+p) \sum_{k=n+p}^{\infty} k a_{k} \leq \mid b[\gamma(p-$ $1)+1] \phi(p)+(p-|b|)[\gamma(n+p-1)+1] \phi(n+p) \sum_{k=n+p}^{\infty} a_{k} \leq|b|[\gamma(p-1)+$ $1] \phi(p)+(p-|b|)[\gamma(n+p-1)+1] \phi(n+p)\left(\frac{|b|[\gamma(p-1)+1] \phi(p)}{(n+|b|)[\gamma(n+p-1)+1] \phi(n+p)}\right)=|b|[\gamma(p-$ $1)+1] \phi(p)+\frac{|b|(p-|b|)[\gamma(p-1)+1] \phi(p)}{(n+|b|)}=\frac{|b|[\gamma(p-1)+1] \phi(p)(n+|b|)+|b|[\gamma(p-1)+1] \phi(p)(p-|b|)}{n+|b|}$ $=\frac{|b|[\gamma(p-1)+1] \phi(p)(n+p)}{n+|b|}$ Hence $\sum_{k=n+p}^{\infty} k a_{k} \leq \frac{|b|[\gamma(p-1)+1](n+p) \phi(p)}{(n+|b|)[\gamma(n+p-1)] \phi(n+p)}=: \delta,(p>|b|)$ which completes the proof of Theorem 3.1.

In a similar manner, by applying the assertion (2.4) of Theorem 2.2 to functions in the class $G_{\eta, \lambda}^{P}(\gamma, b)$,we can prove the following inclusion relationship.

\section{Theorem 3.2. If}

$$
\delta:=\frac{p(|b|-1)+[\gamma(p-1)+1] p \phi(p)}{[\gamma(n+p-1)+1] \phi(n+p)}
$$

then

$$
G_{\eta, \lambda}^{P}(\gamma, b) \subset N_{n, \delta}(h)
$$

\section{Further Neighborhood Properties}

In the last section, we determine the neighborhood properties for each of the following function classes: $J_{\eta, \lambda}^{p, \alpha}(\gamma, b)$, and $G_{\eta, \lambda}^{p, \alpha}(\gamma, b)$ Here the class $J_{\eta, \lambda}^{p, \alpha}(\gamma, b)$ 
consists of $f(z) \in A_{p}(n)$ for which there exists another function $g(z) \in J_{\eta, \lambda}^{p}(\gamma, b)$ such that:

$$
\left|\frac{f(z)}{g(z)}-1\right|<p-\alpha,(z \in U ; 0 \leq \alpha<p) .
$$

Similarly, the class $G_{\eta, \lambda}^{p, \alpha}(\gamma, b)$ consists of $f(z) \in A_{p}(n)$ for which there exists another function $g(z) \in G_{\eta, \lambda}^{p}(\gamma, b)$ satisfying (4.1). Now, using (1.5),(4.1) and Theorem 2.1 ; then $(1.5),(4.1)$ and Theorem 2.2 we can respectively prove the following results:

Theorem 4.1. Let $g(z) \in J_{\eta, \lambda}^{p}(\gamma, b)$. Suppose that

$$
\begin{aligned}
& \alpha:= \\
& p-\frac{\delta(n+|b|)[\gamma(n+p-1)+1] \phi(n+p)}{(n+p)[(n+|b|)[\gamma(n+p-1)+1] \phi(n+p)-|b|[\gamma(p-1)+1] \phi(p)]} .
\end{aligned}
$$

Then $N_{n, \delta}(g) \subset J_{\eta, \lambda}^{p, \alpha}(\gamma, b)$.

Theorem 4.2. Let $g(z) \in G_{\eta, \lambda}^{p}(\gamma, b)$. Suppose that

$$
\begin{gathered}
\alpha:= \\
p-\frac{\delta[\gamma(n+p-1)+1] \phi(n+p)}{(n+p) \phi(n+p)[\gamma(n+p-1)+1]-p(|b|-1)-[\gamma(p-1)+1] p \phi(p)} .
\end{gathered}
$$

Then $N_{n, \delta}(g) \subset G_{\eta, \lambda}^{p, \alpha}(\gamma, b)$.

\section{References}

[1] O.Altimaş,Özkan, H.M.Srivastava, Neighborhoods of a certain family of multivalent functions with negative coefficients, Comput.Math.Appl.47(2004) 1667-1672.

[2] O.Altimaş,Özkan, H.M.Srivastava, Neighborhoods of a class of analytic functions with negative coefficients, Appl.Math.Lett.3(2000) 63-67.

[3] H.M.Srivastava, Halit Orhan, Coeffiecient inequalities and inclusion relations for some families of analytic and multivalent functions,Appl.Math.Lett.20(2007) 686-691.

[4] A.W.Goodman, Univalentfunctions and nonanalytic curves, Proc.Amer.Math.Soc.8(1957) 598-601. 
[5] H.Orhan, E.Kadioğlu, Neighborhoods of a class of analytic functions with negative coefficients, Tamsui Oxford J.Math.Sci.20(2004) 135-142.

[6] H.Orhan, M.Kamali, Neighborhoods of a class of analytic functions with negative coefficients, Acta Math.Acad.Paedagog.Nyházi.(N.S) 1(2005) 5561.

[7] G.Murugusundaramoorthy, H.M.Srivastava, Neighborhoods of certain classes of analytic functions of complex order, J.Inequal.Pure Appl.Math.2(2004) 1-8.

[8] R.K.Raina, H.M.Srivastava, Inclusion and neighborhood properties of some analytic and mulivalent functions, J.Inequal.Pure Appl.Math.1(2006) $1-6$.

[9] S.Ruscheweysh, Neighborhoods of univalent functions, Proc.Amer.Math.Soc.81(1981) 521-527.

[10] H.M.Srivastava, S.Owa(Eds), Current Topics in Analytic Function Theory, World Scientific Publishing Company,S ingapore, New Jersy, London, Hong Kong, 1992.

[11] J.SALAH, M.DARUS, A subclass of uniformly convex functions associated with fractional calculus operator involving caputo's fractional differentiation. Acta. Univ. Apl. 24 (2010) 295-306. 
